\title{
Value Oriented Designing of Healthcare Service Systems
}

\author{
Gilmini Dantanarayana \\ Department of Computer Science \\ Faculty of Science \\ University of Ruhuna \\ Sri Lanka
}

\author{
Prasad Jayaweera \\ Department of Computer Science \\ Faculty of Applied Sciences \\ University of Sri Jayawardenapura \\ Sri Lanka
}

\begin{abstract}
Early identification, complete and correct designing of services are fundamental for any successful e-Business solution in present-day. However, multiparty collaborative environment offering very many diverse services in healthcare context has resulted not only difficulties in requirement elicitation but also multitude of burdens during e-Health service designing and development. Yet another facet that hinders achievement of successful e-Health service deployments is the unavailability of framework that could facilitate systematic alignment between higher level strategic and motivational requirements with lower technical level realizations. This research work is an initiative contributing to get established a framework that could facilitate successful e-Health service deployment while overcoming afore mentioned deficiencies, ensuring semantic and technical service interoperability. A value object classification schema and associated Information Referencing Models has been introduce in this paper that can be serves as theoretical basis for healthcare e-Service Solution industry. The advantage of the proposed modeling guidance is traceability between higher level motivational requirements and subsequent lower level value \& service modeling layers. Draft modeling of an application area based on the proposal has been briefly demonstrated in order to illustrate the potential applicability of the proposed framework.
\end{abstract}

\section{Introduction}

Ever increasing diversity and differentiation due to expansions in service providers, variety of service organizations and constantly created new medical specialties has resulted challenging, complex and highly dynamic environment for e-Health solution developer. Therefore it is along standing needed for a systematic approach to capture highly dynamic and emerging organizational requirements in order to achieve successful e-Health solution meeting stakeholders' needs. Yet another facet that hinders achievement of successful e-Health service solution deployments is the unavailability of framework that could facilitate systematic alignment between higher level strategic and motivational requirements with lower technical level realizations.

Value oriented service identification is one of the proven and current approach that could address the issues introduce above and then to reach successful eBusiness solutions. Complete guidance of value oriented framing of healthcare services is essential even though it is complicated. Different constructs at different levels of modeling layers needed to be represented and specified capturing highly dynamic and complex of business requirements of service solutions. Service oriented enterprise applications are worth addressing in businesses having networked business atmosphere. It is worthwhile to consider on value objects transferred among multiple actors involved in these service offerings in healthcare. Also deciding on associated actor perspectives along with relevant value objects is the foundation for early identification and then to design motivation-aligned e-Services for healthcare industry.

General trading setup in which tangible commodities carrying various forms of values are oftentimes being transferred among collaborating trading parties, they are get completed through simple and straight forward mostly through binary forms of business collaborations. When comparing against general trading setup, however, healthcare domain consists of highly networked collaborating environment where intangible service co-creation and offering occurs through complex service coordination. For instance in curing sub-sector, the healthcare service received by the patient is very much an accumulated with set of services that are being offered by spectrum of curing and caring service providers together in association with different facilitations and coordination supports. Also there are multiple service providers who offer similar but also with varying quality of healthcare 
services and it is expected that a patient could be in a position to obtain required services as per her capacity and wish. When considering these complex situations, it is necessary to get established a unified framework that could facilitate analysis, designing, development and deployment of healthcare services solutions offered on this shared and dynamic environments.

Another issue in this shared environment is due to mandatory and unavoidable very many multiparty service collaborations. These difficulties in integrating heterogeneous healthcare application could potentially result interoperability issues in return as well.

Service Aware Interoperability Framework (SAIF) is an effort focused mainly on achieving working interoperability with the adoption of service oriented architecture. However SAIF has also been criticized in literature [2] from different aspects for issues such as quality, consistency and completeness related.

The work reported here is an approach to model services with a business motivation driven basis using notions of value objects as an attempt to meet issues and deficiencies briefed above. The approach has also being extended by information reference models for healthcare information requirement with the objective of catering needs of Information sub-framework of SAIF as briefed in part B of section III. Also the associations among modeling elements are illustrated by means of meta-models that could also assist in analyzing services identified at early stages of solution development workflow. Our previous research work [11], [12] covered goal oriented value objects classification for healthcare domain and set of guidelines to identify them and apply towards framing services. Further improving our contributions towards more comprehensive service identification approach has been reported in this work.

The rest of the paper is structured as follows. Section II briefs the adopted research methodology. Section III describes related literature study focusing on standard frameworks in healthcare domain as well as modeling approaches in service oriented enterprise solution designing. Section IV gives the overview of VOD framework. Section V and VI, outlines Value Object classification and proposed Value Object Reference Information Modeling in healthcare domain respectively. Next, Section VII illustrates how the proposed approach is applied to identify composition and relationships of components with regard to value objects in healthcare domain and finally, Section VIII concludes with brief evaluation and benefits of the proposed approach.

\section{Adopted Methodology}

We have adopted a research methodology based on Design Science [14] for the development of proposed meta-model based value objects in healthcare. Stepwise approach consists of build, evaluate, theorize and justify the artifacts have been followed. Recommendations from global technology forums and international industry standards have been associated in this work. Development of modeling constructs and Information Referencing Models as facilitation to comprehensive service identification approach and investigate fidelity with real world case studies have been done conforming to the design science guidelines.

\section{Related Work and Research Background}

The value oriented service designing approach for e-Health service solution development proposed in this work has been inspired by global standardizations efforts for technology and healthcare and well-known value modeling contributions.

HL7 [1] being the world largest healthcare standardization consortium and OMG [4] being leading non-profit organization for global technology standardization, their initiative, SAIF [2] has been extensively used as the framing foundation for proposed approach. Derivation of meta-modeling has been done in line with the UMM (UN/CEFACT's Modeling Methodology) [6] meta-modeling recommendations. The following sections brief the major related areas of aforementioned contributions and standards.

HL7 is an organization that develops international standards for IT in healthcare domain initially concerning point-to-point messaging [1]. HL7 being most widely used healthcare standard is a comprehensive framework for exchange, integration, sharing and retrieval of electronic health information supporting clinical practice and the management to deliver and evaluate the healthcare service. Development, adoption, market recognition, utilization and adherence standards are specified in HL7 covering the complete life cycle of businesses.

Object Management Group (OMG) is one of the leading organizations promoting Object Orientated and related IT solution development standards. HL7 together with OMG have proposed SAIF, the leading framework that is intended to guarantee interoperability among healthcare applications. SAIF resulted as an extension for the HL7 and tries to cover several aspects in healthcare service modeling based on working interoperable behavior in this domain [1]. However research analyzing SAIF conclude that it failed to satisfy the principals relative to both architecture and computation behavior in information modeling [2]. SAIF consists of four sub-frameworks; Enterprise Conformance and Compliance Framework (ECCF), Governance Framework (GF), Behavioral Framework (BF), Information Framework (IF). ECCF: ECCF provides a Specification Stack (SS) 
which used to collect testable conformance statements. It allows to assessing the risks throughout entire business. The constructs can be organized according to Reference Model Open Distributed Processing (RM-ODP) [13] viewpoints and Model Driven Architecture (MDA) layers [16]. The major viewpoint of ECCF is achieving interoperability between different actors. Governance Framework (GF): This framework basically describes how the internal HL7 governance could be achieved. Also it is about the relationships between HL7 and other organization standards, relationship between standards and government regulations and policies and governance within interoperable parties. Business policies and rules are formulated based on ECCF with focusing the content in $\mathrm{BF}$ and IF introduced next. This framework deals with authorization power, verifies performance and manages configuration baseline.

Behavioral Framework (BF): The set of artifacts that covers the dynamic semantics support Working Interoperability and also it describes accountability perspectives concerning responsibility assignments.

Information Framework (IF): This refers static semantics and existing information framework artifacts. This work addresses service identification in healthcare sector with main focus on to strategic and motivational perspectives of actors. That is to facilitate technology neutral service identification that in return could also assist in lower level technical service realizations. The above four sub frameworks can be mapped into the popular three levels of modeling layers; Why, What and How aspects. ECCF and GF involves strategic and motivational levels of the enterprise which specifically gives "why" and "what" aspects of the business while BF and IF mainly focusing "how" and "what" aspects of the business. However the work reported here is in line mainly with two sub frameworks ECCF and GF that cover "why" and "what" aspects of the organization with an objective of maintaining semantic interoperability.

Goal oriented value object classification and framing services using value activities conform the goal modeling and value modeling standards [11]. Goal oriented value object classification has been developed using business motivation model [4] as goal modeling methodological foundation and then the work also introduced phases of applying the proposed classification towards extending business value modeling in terms of $\mathrm{e}^{3}$ Value models [3]. Therefore our previous work facilitated the systematic guidance to align these "why" and "what" aspects of the healthcare service organization.

Adopting this work towards more comprehensive service identification by identifying the elements and defining associations among them in terms of metamodels are the central focus in this work.
When semantics of a particular notation of modeling is defined in the form of a model such as a UML class diagram, it is called a meta-model [6]. The semantics are defined in meta-models in order to facilitate modeling elements in a model instance. The essentials included and the irrelevances excluded in a system or software is captured through these types of meta-modeling [7]. The way of new characteristics and constraints are assigned to a meta-class by extending the semantics, with the use of stereotypes.

RIM is one of the critical components in HL7 v3 model consists of generic level of classes based on Unified Meta Modeling Language that describe the information perspective of healthcare. Further these resulted RIM models are technology-neutral models and can be utilized as documents, data for services and messages covers HL7 domain of interest [15]. However the RIM models do not depend on any higher level motivational requirements or value modeling requirements, thus it is hard to figure out the relationship between information modeling with higher level design requirements, i.e. in other words hard to achieve bi-directional traceability between enterprise modeling layers. Therefore this work tries to adopt RIM models based on goal oriented value objects in Healthcare (HC) domain.

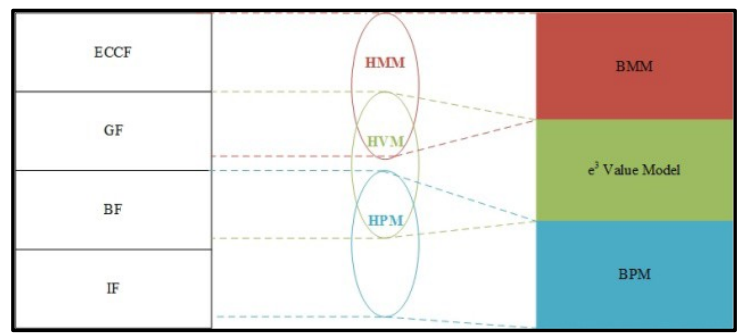

Figure 1. VOD framework bridging SAIF SubFrameworks and Enterprise Modeling Layers

The philosophy of business enterprise modeling followed three perspectives of the business, goals/motivations, values and processes. Similarly HC solution designing development could also be formalized according to this popular approach. However still there is lack of guidance for high level architectural model specifically addressing service designing in $\mathrm{HC}$ sector while ensuring alignment with global standards. In this study we propose the three layer model that consist of HMM (Healthcare Motivational Modeling), HVM (Healthcare Value Modeling) and HPM (Healthcare [Service] Process Modeling) as a systematic approach to overcome many drawbacks identified in this domain as depicted in Figure 1.

Value Object Design (VOD) framework that has been proposed consists of three higher level modeling layers as briefed as follows. 
Initially it is necessary to focus on to the motivations of the actors in healthcare service collaborations in order to design successful solution. There are several actor categories with varied motivational requirements and aspects involved in HC service offering settings. Analysis of these motivational aspects and collaborations among different actors would result three actor perspectives in $\mathrm{HC}$ as identified for our framework.

Primary Perspective: When considering healthcare scenarios, it is notable that the ultimate customer to be considered central is patient and we refer patient's perspective as Primary Perspective in motivation modeling effort in HC.

Governing perspectives: When carefully analyzing healthcare service collaborations, it is evident that there are Governing Collaborations (between Physicians and Patients) that enact, control and coordinate all the other Auxiliary Collaborations between other supportive healthcare service providers such as Medical Laboratory Technicians, Pharmacists, Radiologists, etc. Therefore, we recommend also considering these two sub perspectives; Governing Perspective and Auxiliary Perspective also during motivation modeling efforts.

Auxiliary Perspectives: Besides governing perspectives, other collaborations between different stakeholder categories are to offer some assistance/facilitation services to the major treatment services towards patient. For Example, Medical Laboratory Technician offer medical report to patient when he was given formal order by authorized physician, i.e. governing actor. Further pharmacist gives the medicine to patient only for authorized prescription by physician, i.e. again from governing actor. Therefore it is difficult to complete the major healthcare services in absence of these assistant services done through auxiliary collaborations.

In general $\mathrm{HC}$ objects are considered to be carrying different types of values. Also notion of 'value' is more important to derive service designing because it defines the 'what' view point of the business context in terms of choreography of value exchanges supporting different business contexts.

Healthcare values are carrying/integrated with resources occurred in varied forms such as "rights", "custody", "evidence documents" or "instruction to third party" etc.. [3]. Most of the objects exchanged among HC actors are composed with one or more of these different types of values. However it is important to identify the types of values attached with HC objects since they influence correct and complete designing hence ensuring effectiveness of resulting service system.
A right of an exchanged resource means the resource-holder is entitled to use/consume the resource. But it could occur in varied forms of rights for instance, right to use with ownership or right to use without ownership. An example of this situation is when patient transfers money for $\mathrm{HC}$ service organization, the organization receives the ownership with right to use/consume money.

Physical access to an object is limited in some situations and custody could be referred as the form of limited access to the object. For instance, HC laboratory testing sample collection centers only responsible to keep in the custody for sample collected until they deliver to the main medical laboratory testing center.

Evidence documents are most important form of value used in healthcare objects where intangible value creations occurred. For instance, in many situations a proof of the treatment carried out and healing situation in the form of evidence documents. For instance, when a specific operation is performed on a patient, an evidence document would be main acceptable way of proving the task carried out successfully.

Auxiliary services such as medical laboratory testing or giving prescribed medicine to patient is only carried out according to the instructions given by relevant authority as referred governing party in this work(e.g. physician). They can be considered as instructions to third party because the patients are not allowed/authorized to request the service directly from these auxiliary actors without formal order/s from governing actor/s.

The central point focusing in this research work is HC values which provide a basis for Value Object classification and Reference Information Models discussed in the sections V and VI respectively.

\subsection{VO Service Process Design in HC}

A systematic healthcare service process designing is a must to be adopted in order to meet higher motivational requirements of the major actor categories introduced in VOD framework. In addition to the $\mathrm{HC}$ value object classification, HC service process activities classification has been defined [12]. The classification consists of Service Acquisition Value Activity (SAVA), Decision Making Value Activity (DMVA), Directing Value Activity (DVA) and Service Supportive Value Activity (SSVA) which assisted to cover the major service activities in the HC context. These service activities are assisted in completion of service process design by initiating motivational driven value objects. 
Further few meta models have been proposed in [12] to demonstrate the relationship among actors, Value Objects and Value Activities facilitate service designing through the three layers explaining in section $\mathrm{A}, \mathrm{B}$ and $\mathrm{C}$.

\subsection{Value Object Classification}

A Value Object classification has been proposed for VOD Framework [11] by considering different viewpoints mentioned in section IV and considering behavioral aspects when exchanging healthcare value objects.

According to goal oriented value objects classification for healthcare [11], four types of categories, Intrinsic Value Object (IVO), Co-created Value Object (CVO), Recurrent Value Object (RVO) and Affirmative Value Object (AVO) have been proposed is briefed as follows.

Intrinsic Value Object (IVO): IVO refers the object need to transfers in order to receive the entire service and receiving party of IVO has no immediate interest other than personal consumption in corresponding to concerned healthcare collaboration.

For instance, the patient fee, a payment is transferred to the admission office by a patient. The received payment will not be transferred via another value transfer in the healthcare collaborations within the case under consideration.

Recurrent Value Object (RVO): Value objects that are repeatedly used in many different value transfers of a value model referred as RVO.

Example: In this case study, same set of medical reports of a patient is transferred among several actors; from medical laboratory to OPD, from OPD to ward and from ward to operation-theater, etc. It may be important and relevant to ensure the health condition of a patient prior to any medical/surgical treatment related activities. Therefore, medical reports are being transferred recursively in order to provide/share necessary patient's health state information.

Co-created Value Objects (CVO): These are the value objects that could also be transferred via value transfers but they could also be accesses or/and add value separately or simultaneously by different value actors.

There are situations where multi parties involving with creating same value objects. Responsibility of creating and accessing of single value object is with more than one actor. Another special feature could be the part of the object created by one actor becomes a value to another actor at later time. These special property requirements could be defined as co-created value object and could be mapped accordingly.

In the case, patient's health records are transferred as patient referral from the admission office to the ward and being updated continuously, same records are accessed and updated by consultants and house officers of a ward and also by operation-theater personnel as well.

Patient information creates different values for many actors in healthcare context. For instance personnel information created by admission office is later needed by people at operation-theater for the purpose of contacting his relatives. Another situation may arise when the surgeon needs to find the past information regarding the patient's health state, he can access the same value object and the information created previously by someone else.

Affirmative Value Objects (AVO): These are kind of affirmative value objects those in occasions composed with other regular value objects, mainly for the authorization and for the verification purposes. These AVOs are to be transferred together with other regular value objects.

In the case study for instance certification of issuer in medical reports, certified surgery results and authorized referral statements should be signed by an authorized healthcare service provider. Unless verified with a signature/endorsement they may not carry any value for subsequent and necessary actions due to policy/regulatory concerns in a particular governing setup.

\section{Healthcare Reference Information Modelling}

Services or goods are exchanged between actors in business environment via collaborations as Value Objects. Value Objects exchanges within and between enterprises can be used as the basis to decide upon the services of an enterprise and they could also provide a mechanism to coordinate them [8] [9][10].

Further the work proposed in [11] stated a set of guidelines explaining how they can be used in assisting service identification with respect to three actor perspectives; governing, primary and auxiliary has been described as briefed in section IV-A. Value Activities identified with respect to actor perspectives and combination patterns of these two phenomena can be analyzed and represented by means of UMM modeling methodologies [12].

Further analysis of distinct properties of CVO and RVO provides the way to propose referencing information modeling initiative accordingly as below. A distinguishing characteristic in RVO is it always associates with a flow of activities while CVO always considers different components/portions of single value objects by responsible actor category. CVOs are not merely created through a flow of activities one after the other instead it enables cocreations and allows accessibility to a group of authorized/contributing actors.

Referencing Information Models associate with the VOs are creditable to consider because it directly related to mapping more detailed service designing requirements. Therefore the following illustrations 
provide assistance to identify and design these different types of VOs more significantly together with referencing information compositions.

\subsection{Recurrent Value Object Reference Information Model(RVO RIM}

The relationship among specific information modeling elements of Recurrent Value Objects can be depicted as in the Figure 1.

RVO results from certain RVO information process consists of set of related value activities in a chain. During this chain of activities, records of current state as well as accumulated workflow states are considered important. Current state refers a snapshot of present activity while accumulated states refer history of all completed activities so far for a treatment case. Each of these value activities may affect to produce some specific status. The current activity may be one value activity among defined value activities of value chain. Also it is necessary to keep track about the actor who is responsible for each of these value activities. Also there are situations where different actors involve for disparate activities or same actor may carry out multiple activities. In some situations current status may represent composite status referred set of different types of areas.

The Recurrent Value Object Referencing Information Model ( $R V O_{R I M}$ ) can be illustrated with case studies based on RVOs; prescriptions, medical reports as explained in the Section IV. CVOs are value objects that could be access or/and add value separately or simultaneously by different value actors [11]. Meta model of Co-created Value Object Referencing Information Model $\left(C V O_{R I M}\right)$ can be represented as in figure 2.

CVO consists of several components/portions and each created and then mostly manipulated by specific actor category. Single actor or set of actors may responsible for each of such a component/portion. Responsibility may be dealt with creation of these pieces and accessibility assignment on them. Some components/portions of these value objects may be shared among different actor categories. The relationships and dependencies among these pieces can be identified and represented as shown in the Figure 2.

\subsection{Application of RIMs}

In this section, a brief illustration of application on proposed healthcare referencing Information models has been given to the right.

\subsection{Application of $\mathrm{RVO}_{\mathrm{RIM}}$}

The application of the $\mathrm{RVO}_{\mathrm{RIM}}$ can be explained using existing case of RVOs in real world healthcare scenarios. Therefore, this project sought to investigate and report on the implementation of the dialogue and its culture in the educational context of a specific university textbook on religious education. Many aspects were addressed that relate to the culture of dialogue between the academic staff and students within the Saudi university, such as the concept of dialogue, the community's values and its virtues, the nature of Saudi society, the university textbooks used for religious education, and the university environment. The initial indicator showed that it was possible to develop the educational textbook to accommodate the culture of dialogue. But the main point was to find how this should be done in the context of Saudi culture, its higher education policy, the university educational activities and regulations, and the relations between the academic staff and students within the classrooms.

The application of the $\mathrm{RVO}_{\mathrm{RIM}}$ can be explained using existing case of RVOs in real world healthcare scenarios. Medical Laboratory Report of a patient, RVO could be used to illustrate the application of RVORIM.

RVO : X-Ray report (Swallow/ Barium meal test)

Value chain: Barium Meal Testing Process

Value Activities : Receive Barium Meal Test Order (RBMTO), Advise for Preparation (AP), Treat with Barium (TB), Get X-rays (GX), repeat the test (RT), Produce Test Results (PTR), Interpret Test Results (ITR), Issue Final Test Report (ITR)

\section{Status : : Current Value Activity}

Eg : Get X-rays (GX)

Actor : Actor assigned for the each activity (Clerical staff, Radiologist, Doctor, technician, etc)

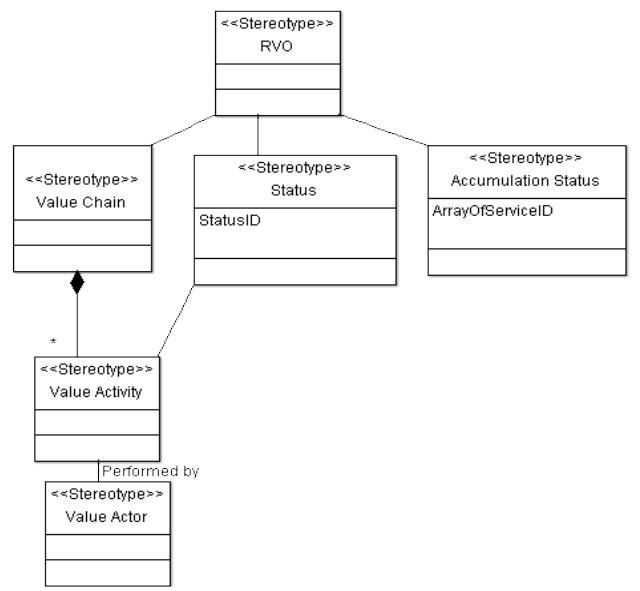

Figure 2. RIM for Recurrent Value Object 


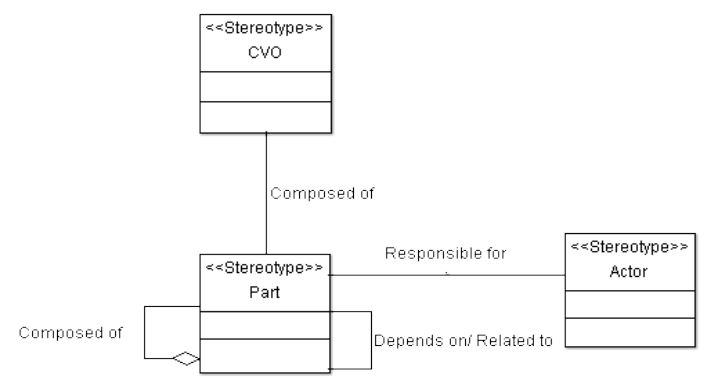

Figure 3. RIM for Co-created Object

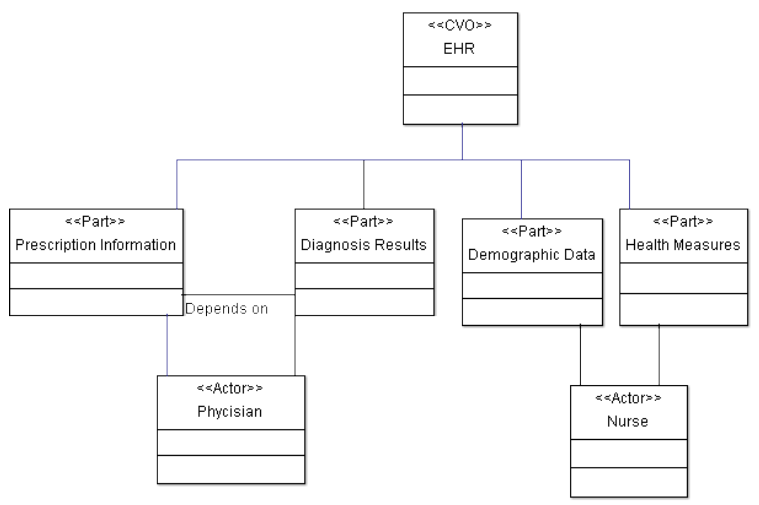

Figure 4. RIM for Co-created Value Object as applied to EHR

Accumulation Status: Activities from Receive Barium Meal Test Order (RBMTO) to Treat with Barium (TB) would be the Accumulation Status if the present status is Get X-rays (GX).

Therefore the elements of the Blood Test Report generation could be identified as above when applying with meta-model shown in Figure 1.

There are several actor levels in Medical Laboratories involved for these activities. For instance clerical staff, MLT, and assistants to MLT are few types of actors responsible for these services in medical report provisioning. Status explains the present value activity carried out among these activities of medical testing process.

There are varied types of medical reports available and it is needed to define sequence of activities for corresponding test report with responsible actors for each defined activity. Laboratory professionals are specially assigned for the functions they perform with certification for their position. For instance, supervisory roles, oversee the testing being performed in the laboratory.

According to this approach, $R V O_{R I M}$ facilitates the way to identify set of activities to be performed in order to provide specific RVO, with actor assignments. Further it assists the ways to keep track of status summary and current status of the service.
The model depicted in Figure 3. gives an example application of reference information model of the CVO. CVO is built by set of responsible actors by adding different values to same object. Electronic Health Record (EHR) is a CVO that has following information modeling elements. The composition of CVO consists of demographic data, Set of health measures, diagnosis results, and prescription information, etc.

Demographic Data: The patient's name, age, ethnicity, race, language are some demographic data of patient. Demographic data is important with some essential/mandatory component composing EHR of a patient. An actor, clerk may be the responsible person for create most of this data module.

Health Measures: Body Mass Index (BMI), Blood Pressure, Temperature, Weight and Height of the patient are some of the health measures needed to record in order to understand the patient health status. Responsibility of completing these data could sometimes be assigned to nursing staff.

Diagnosis Results: Depending upon certain conditions of the patient it is needed to record diagnosis results refers the decisions taken by responsible person, maybe physician to the EHR.

Prescription Information: The medicine ordered to the patient, sometimes related to or dependent on another component of EHR, for instance recorded diagnosis results or measurements such as patient's weight.

The application $C V O_{R I M}$ exemplified in Figure 3. gives how the parts should be co-created in the value object, EHR. Value creation of this object is important aspect to interpret the composition of it in terms of part with actor and the activity involved and dependencies among them.

\section{Conclusion}

Importance of getting established a unified framework that could facilitate successful e-Health service solution development and deployment ensuring semantic and technical service interoperability has been highlighted extensively in literature. The work reported here introduced a contribution in an endeavor to develop a complete and sound value oriented service designing framework. Besides modeling and designing higher motivational requirements to be realized on technologies, the importance of addressing value oriented modeling layer was illustrated in detailed. Value oriented modeling layer mainly consisting Value Objects and Value Activities performed by Value Actors and further these layers that serves as an intermediate layers between higher level motivation/goal modeling layer and lower level service designing technological realization layer. Yet another commendable contribution is the facilitation 
to bi-directional traceability between these modeling layers that designers could be achieve with the adoption of the proposed framework.

In order to achieve the semantic interoperability between healthcare service applications, the fundamental is the Information Reference Model which has been introduced in association with relevant Value Object category. With this value oriented intuition e-Healthcare service designers and developers are with necessary details and guidance in their technological solution development while ensuring the utmost important strategic and policy level compliance and conformance.

However complete and empirical evaluation on the proposed value oriented designing framework is in the agenda of further work of this research with the objective of fine tuning and meet completeness.

\section{References}

[1] G. Eason, B. Noble, and I. N. Sneddon, "On certain integrals of HL7 Architecture Board (ed.): HL7 Service Aware Interoperability Framework: Canonical Version, Release 1, http://www.hl7.org/documentcenter/ (Access Date : 27 November, 2014)

[2] L. Landgrebe and B. Smith.,) "The HL7 Approach to Semantic Interoperability”, ICBO: International Conference on Biomedical Ontology, July 2011.

[3] H. Martin, J. Paul, P. Erik and Z. Jelena, "Value and Goal Driven Design of E-Services", IEEE International Conference on e-Business Engineering (ICEBE'07), pp. 295-303, 2007.

[4] Object Management Group, Inc.: Business Motivation Model (BMM), http://www.omg.org/spec/BMM (Access Date: 27 November,2014)

[5] J. Gordijn, and H. Ackermans, "Designing and evaluating e-business models", IEEE Intelligent Systems, vol. 16, no. 4, pp. 11-17, 2001.

[6] L. Shan and H. Zhu, "Semantics of metamodels in UML”, Theoretical Aspects of Software Engineering, 2009. TASE 2009 IEEE, pp. 55-62, July 2009.

[7] G. T. A. Guedes and R. M. Vicari, "Applying and Validating a UML Metamodel for the Requirements Analysis in Multi-Agent Systems: The AME-A Case Study", SEKE , pp. 746-751, 2011

[8] B. Andersson, P. Johannesson and J. Zdravkovic, "Aligning goals and services through goal and business modeling”, Information Systems and eBusiness Management, March 2009, vol. 7, Issue 2, pp. 143-169

[9] J. Gordijn and M. Petit, "Understanding Business Strategies of Networked Value Constallations Using Value and Goal Modeling”, proceedings of 14th IEEE International Conference on Requirements Engineering, 2006.

[10] Z. Baida, J. Gordijn, H. Saele, H. Akkermans, A. Morch, "An Ontological Approach for Eliciting and Understanding Needs in e-Services" Advanced Information Systems Engineering - CAiSE05, vol. 3520, pp. 400-414, 2005.

[11] G. Dantanarayana, C. Wickramage and P. Jayaweera, "Goal Oriented Value Object Classification for Healthcare Service Development”, Proceedings of the Fifteenth International Conference on Informatics and Semiotics in Organisations, May 2014.

[12] G. Dantanarayana, C. Wickramage and P. Jayaweera, "Framing Services based on Value Activities in
Healthcare” , Proceedings of the International Conference on Advances in ICT for Emerging Regions (ICTer2014)

[13] Vallecillo, A. RM-ODP: "The ISO reference model for open distributed processing". DINTEL Edition on Software Engineering, 3, 66-69, 2001.

[14] Hevner, A., S. March, J. Park, and S. Ram (2004) Design science in information systems research,MIS Quarterly 28 (1), pp. 75-105.

[15] Hasman, A. (2006). HL7 RIM: an incoherent standard. In Ubiquity: Technologies for Better Health in Aging Societies, Proceedings of Mie2006(Vol. 124, p. 133)

[16] OMG, M. (2003). Guide Version 1.0. 1. Object Management Group, 62, http://www.omg.org/mda/ (Access Date:16 January, 2015 